\title{
Experimental Eavesdropping Based on Optimal Quantum Cloning
}

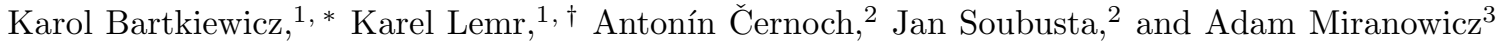 \\ ${ }^{1}$ RCPTM, Joint Laboratory of Optics of Palacky University and Institute of Physics \\ of Academy of Sciences of the Czech Republic, Faculty of Science, Palacky University \\ 17. listopadu 12, 77146 Olomouc, Czech Republic \\ ${ }^{2}$ Institute of Physics of Academy of Science of the Czech Republic, \\ Joint Laboratory of Optics of PU and IP AS CR, \\ 17. listopadu 50A, 77207 Olomouc, Czech Republic \\ ${ }^{3}$ Faculty of Physics, Adam Mickiewicz University, PL-61-614 Poznań, Poland
}

\begin{abstract}
The security of quantum cryptography is guaranteed by the no-cloning theorem, which implies that an eavesdropper copying transmitted qubits in unknown states causes their disturbance. Nevertheless, in real cryptographic systems some level of disturbance has to be allowed to cover, e.g., transmission losses. An eavesdropper can attack such systems by replacing a noisy channel by a better one and by performing approximate cloning of transmitted qubits which disturb them but below the noise level assumed by legitimate users. We experimentally demonstrate such symmetric individual eavesdropping on the quantum key distribution protocols of Bennett and Brassard (BB84) and the trine-state spherical code of Renes (R04) with two-level probes prepared using a recently developed photonic multifunctional quantum cloner [K. Lemr et al., Phys. Rev. A 85, 050307(R) (2012)]. We demonstrated that our optimal cloning device with high-success rate makes the eavesdropping possible by hiding it in usual transmission losses. We believe that this experiment can stimulate the quest for other operational applications of quantum cloning.

PACS numbers: 42.50.Ex, 03.67.Ac, 03.67.Dd, 03.67.Lx
\end{abstract}

During the last decades, there has been much interest in secure quantum communication [1, 2]. Quantum key distribution (QKD) devices (apart from quantum metrology, random number generators and adiabatic computers based on quantum annealing) are arguably the only second-generation quantum technologies providing commercially available applications of quantum information and quantum optics up to date [3]. The security of QKD follows from Heisenberg's uncertainty relation or, equivalently, the no-cloning theorem. However, QKD can be secure only below some level of noise that unavoidably occur in any physical system. Therefore, security bounds of QKDs are expressed in terms of tolerated losses or noise.

For QKD to be secure Alice and Bob must operate on single photons; hence, they need a single-photon source (SPS). SPSs are usually implemented as a weak coherent pulse of light [1]; thus, QKD are prone to photon-number splitting attacks. This attack can be circumvented by, e.g., using decoy states [4] or heralded SPS instead of weak coherent pulses. Since there are no lossless channels, if the eavesdropper (Eve) is equipped with a proper cloning machine mimicking the lossy channel then she can clone (a part of) the state sent by Alice, while hiding her presence in usual transmission losses.

Recent proposals of applications of quantum cloning 5 ] range from quantum cryptography [6] and quantum metrology 7] to nonclassicality tests in microscopicmacroscopic systems [8] and, even, proposals related to quantum experiments with human eyes $[9]$.

\footnotetext{
*Electronic address: bartkiewicz@jointlab.upol.cz
}

${ }^{\dagger}$ Electronic address: k.lemr@jointlab.upol.cz
In this Letter, we experimentally demonstrate the usefulness of cloning for quantum cryptoanalysis, i.e., for the eavesdropping of QKD over noisy quantum channels.

There are a number of well-known QKDs including the famous BB84 of Bennett and Brassard [10] based on mutually unbiased bases and the biased-bases R04 of Renes [11]. Attacks on those protocols can be classified as individual (or incoherent) and coherent (including joint and collective) [1]. Every attack can be imagined as follows: Eve sends a photon (probe) prepared in some polarization state which interacts with a photon sent by Alice, then Eve sends a photon to Bob and performs a measurement on her probe (she might wait until the key sifting process is over). Recently, attacks on QKD were proposed exploiting technological loopholes rather than the limits imposed by physics [12, 13]. However, in this Letter we analyze the physical bounds on the security of the QKDs.

We focus only on individual attacks on the two QKDs assuming that Eve waits until Alice and Bob complete key sifting and then performs her measurements. This kind of attack requires that Eve has access to quantum memory (QM) in order to store her probes during the key sifting, but does not require Eve to perform a coherent measurement on many photons at a time. Such satisfactory memory has not been invented yet; however, recent encouraging results [14] carry the promise of realizing good QMs in near future. Moreover, in our opinion, coherent multi-qubit readout may require an additional technological leap. For clarity of presentation we focus only on trine-state R04 and BB84. Nevertheless, our approach can be used for analyzing generalizations of those protocols. By referring hereafter to R04 we mean its 


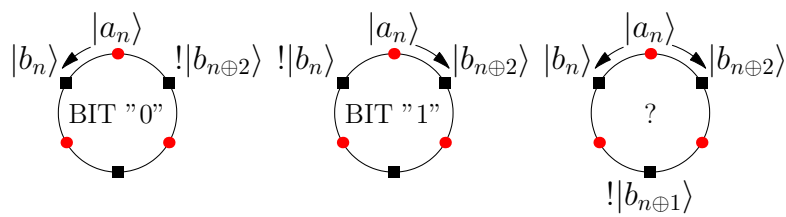

FIG. 1: (Color online) Diagram describing R04 11]. Alice (Bob) publicly agrees beforehand to send (measure) one of the trine states marked by red (black) dots, respectively. Both agree that the clockwise (anticlockwise) sequence of their states corresponds, e.g., to bit 1 (0). Bob publicly informs Alice what he has not measured (marked by an exclamation mark). Alice ignores the inconclusive cases (and informs Bob about them). In the other two cases, Alice and Bob obtain the same bit value.

trine-state version.

It is known that the acceptable quantum bit error rate (QBER), i.e., the ratio of the number of wrong qubits to the total number of qubits received, is 15\% [15] for BB84 and $16.7 \%$ [1] for R04 assuming an individual attack with a four-level probe and that Eve does not wait for the key sifting. These QBER bounds could suggest that R04 is more robust to eavesdropping than BB84. However, it was shown that, by assuming one-way communication between Alice and Bob, BB84 is unconditionally secure if $\mathrm{QBER} \leq 11 \%$ [16], while R04 if QBER $\leq 9.85 \%$ [17]. On the other hand, if Eve waits for the key sifting process to finish, BB84 is secure if QBER $\leq 14.6 \%$ [18] (or 15\% [19] for the two-level probe), while the corresponding QBER bound for R04 is unknown to our knowledge. Nevertheless, in this Letter we show that the QBER bound for R04 and BB84 is $16.7 \%$ for the optimal cloning attack with a two-level probe.

The algorithm for the cloning-based eavesdropping investigated in our Letter reads as follows: (i) Eve plugs a cloning machine together with QM into the quantum communication channel between Alice and Bob. (ii) Alice sends one of the states used in BB84 or R04. (iii) Eve intercepts the state and prepares two noisy copies. This cloning introduces losses. (iv) Eve sends one copy to Bob and keeps the other copy in QM. (v) Bob measures the received copy. (vi) Alice and Bob publicly perform key sifting. (vii) Eve performs positive-valued measures (POVMs) on each of the stored qubits to guess the bit value that was obtained by Alice and Bob simultaneously. She assigns corresponding bit values to her measurement outcomes. The steps performed by Eve are discussed below (for additional details see Ref. [20]). Since, in our experiment, we do not have access to QM we simulate it by performing a reconstruction of the two-qubit density matrix shared by Eve and Bob and later by projecting Bob's part of the state onto one of the bases used in QKD. The reduced density matrix describing Eve's qubit is assumed to be stored in QM.

Symmetric attacks on QKD can be performed by using a multifunctional optimal quantum cloner (OQC) [1]. In R04 [11] (explained in Fig. 1) Alice sends one of the three equally separated equatorial qubits $\left|a_{n}\right\rangle=\mathcal{N}[|H\rangle+$ $\exp (i 2 n \pi / 3)|V\rangle]$ and Bob detects $\left|b_{n}\right\rangle=\mathcal{N}[|H\rangle+$ $\exp (i 2 n \pi / 3+i \pi / 3)|V\rangle]$, where $n=0,1,2$ and $\mathcal{N}=$ $1 / \sqrt{2}$. Since all the states used in R04 (and also in BB84) are on the equator of the Bloch sphere (say $x y$ plane), we require that Eve's action causes the Bloch sphere of the qubit received by Bob to shrink uniformly in the $x y$ plane (qubit's purity decreases) so that her presence cannot be easily detected. Thus, the density matrix of Bob's qubit reads as $\rho_{B}=\frac{1}{2}\left[\mathbb{1}+\left(\hat{\eta}_{B} \vec{r}_{B}\right) \cdot \vec{\sigma}_{B}\right]$, where the Bloch-sphere shrinking is described by matrix $\hat{\eta}_{B}=\operatorname{diag}\left(\eta, \eta, \eta_{\perp}\right)$, where $\eta\left(\eta_{\perp}\right)$ is the shrinking factor in the $x y$ plane ( $z$ direction), $\vec{r}$ is the Bloch vector of the initial qubit, and $\vec{\sigma}=\left(\sigma_{x}, \sigma_{y}, \sigma_{z}\right)$ is a vector of Pauli's matrices. Our OQC [1] provides the following shrinking factors $\eta=2 \sqrt{p} \Lambda \bar{\Lambda}$ and $\eta_{\perp}=\Lambda^{2}+\bar{\Lambda}^{2}(p-q)$, where $q+p=1$ and $\Lambda^{2}+\bar{\Lambda}^{2}=1$ assuming that $p, q, \Lambda, \bar{\Lambda} \in[0,1]$, where $p$ is the asymmetry parameter of the clones and $\Lambda$ is the cloning "strength" since it affects the purity of the clones (related to the shrinking factors) in the same way. In our experiment we fix values of $p$ and $\Lambda$ by adjusting polarization sensitive filtering in BDAs (see Fig. 2] and Ref. [20]). Moreover, for Eve's probe we obtain $\hat{\eta}_{E}(p, \Lambda)=\hat{\eta}_{B}(q, \Lambda)$. This operation is similar to the one of the mirror phase-covariant cloner [1, 22]. The difference depends on $p$ which implies that the states of Eve and Bob have different fidelities with respect to the states sent by Alice. Furthermore, the fidelity of Bob's qubits is $F_{B}(p, \Lambda)=(1+2 \sqrt{p} \Lambda \bar{\Lambda}) / 2$, whereas Eve obtains $F_{E}(p, \Lambda)=F_{B}(q, \Lambda)$. The unitary cloning transformation reads as $|H\rangle_{A} \rightarrow[\Lambda|H, H, 0\rangle+\bar{\Lambda}|\psi(p), 1\rangle]_{B, E \text {,anc }}$ and $|V\rangle_{A} \rightarrow[\Lambda|V, V, 1\rangle+\bar{\Lambda}|\psi(q), 0\rangle]_{B, E, \text { anc }}$, where $|\psi(p)\rangle=$ $\sqrt{p}|H, V\rangle+\sqrt{q}|V, H\rangle$. The resulting state shared by Bob and Eve is obtained by tracing out the ancilla, which in our experiment corresponds to random switching between $H$-and $V$-polarized photons used by Eve as probes. For $p=\Lambda^{2}=1 / 2$ the OQC becomes the symmetric $1 \rightarrow 2$ phase-covariant cloner [23], which for BB84 causes $\mathrm{QBER}=1-F_{B}=14.6 \%$. Moreover, for $p=1 / 2$ and $\Lambda^{2}=2 / 3$, the OQC becomes the universal cloner [24]. We assume Eve's probe to be a qubit, while the most general approach requires the probe to be a four-level system. Our restriction is valid if two-photon interactions [1] are only used for the eavesdropping.

Optimal eavesdropping strategy. - Eve knows the initial state of her photon as her OQC performs conditional operations [1, 20], where the asymmetry is implemented by introducing additional losses [25]. However, Eve, to optimize her attack on R04, must choose the optimal strategy for distinguishing between Bob's measurement results $b_{n}$ and $b_{n \oplus 1}$ given that Alice sent $a_{n}$ and $a_{n \oplus 2}$, respectively, where $\oplus$ stands for sum modulo 3 . While restricting Eve's readout to the von Neumann's measurements we found the optimal ones maximizing Eve's information (this follows from the symmetry of the shrinking factors) to be equivalent to Helstrom's measurements [26] discriminating between states $\left|b_{n}\right\rangle$ and $\left|b_{n \oplus 1}\right\rangle$ (or $\left|a_{n}\right\rangle$ and $\left.\left|a_{n \oplus 2}\right\rangle\right)$ independent of the values $\Lambda, p$, and the initial 


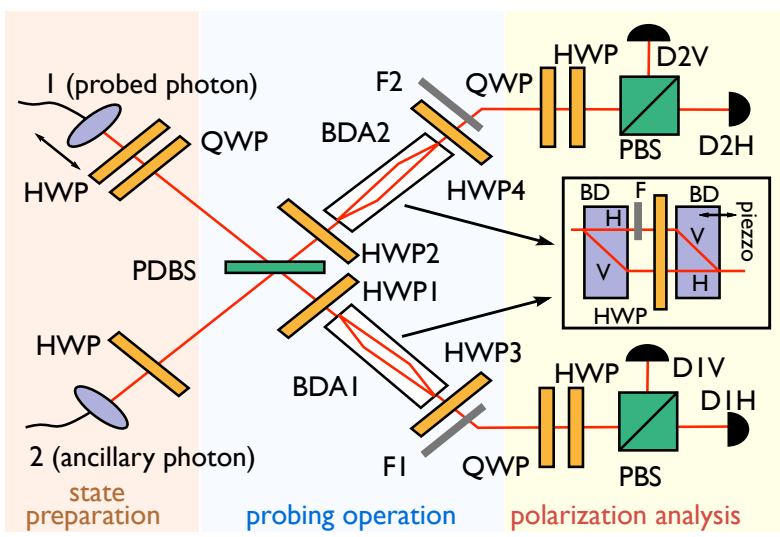

FIG. 2: (Color online) Experimental setup as described in the text. States of the probed and ancillary photons are prepared with half-wave (HWP) and quarter-wave (QWP) plates. The photons overlap on the polarization-dependent beam splitter (PDBS) and undergo polarization-sensitive filtering in the beam divider assemblies (BDAs). Each BDA (see figure inset) consists of a pair of beam dividers (BDs), a neutral density filter $(\mathrm{F})$, and a half-wave plate (HWP). The tomography of the two-photon state is accomplished by means of the HWPs, QWPs, polarizing beam splitters (PBDs), and single-photon detectors (D).

state of the probe. Thus, Eve's measurement is a projection on equatorial qubits of phase $2 n \pi / 3+\pi / 6+m \pi$ $(2 n \pi / 3+5 \pi / 6+m \pi)$ if Bob's message (see Fig. 11) is ! $\left|b_{n}\right\rangle$ $\left(!\left|b_{n \oplus 2}\right\rangle\right)$, where $m=0,1$ is Eve's bit value. For BB84, Eve uses the measurement as Bob.

For the measurements we calculated [20] the mutual Shannon information $I_{X, Y}$ between the three users, where $X, Y$ stand the initials of the corresponding parties. Next, we calculated the secret-key rate (i.e., the lower bound on the distilled key length per number of the sifted-key bits) $R=I_{A, B}-\min \left(I_{A, E}, I_{B, E}\right)$ [27] as a function of $\Lambda$ and $p$. Finally, we found the optimal cloning attack by maximizing $I_{A, B}$ for $R=0$. The results of our theoretical analysis, as summarized in Fig. 3 , imply that the optimal (cloning restricted) two-level-probe individual attack on $\mathrm{R} 04$ yields $\mathrm{QBER}=16.7 \%$ for $\Lambda^{2}=4 / 11$ and $p=4 / 7$. Our results for the analogous strategy for BB84 are shown in Fig. 3, where the best attack yields $\mathrm{QBER}=16.7 \%$ for $\Lambda^{2}=1 / 3$ and $p=1 / 2$. The QBER depends on the fidelity of cloning $\left[\mathrm{QBER}=1-F_{B}\right.$ for $\mathrm{BB} 84$ and $\mathrm{QBER}=4\left(1-F_{B}\right) /\left(5-2 F_{B}\right)$ for R04], while the information extracted by Eve depends both on the fidelity (as does $I_{A, E}$ ) and the entanglement of clones (correlations between Bob's and Eve's qubits). Thus, the optimal attack must balance these two quantities to provide $R=0$ for a given $I_{A, B}$.

Experimental aspects of the eavesdropping. - In order to implement the cloning attack, we employed the experimental setup (see Fig. 2), which consists of three main parts: the source of photon pairs, the cloner and the twophoton polarization analyzer. Spatially-separated photon pairs of $\lambda=826 \mathrm{~nm}$ wavelength are created in non-

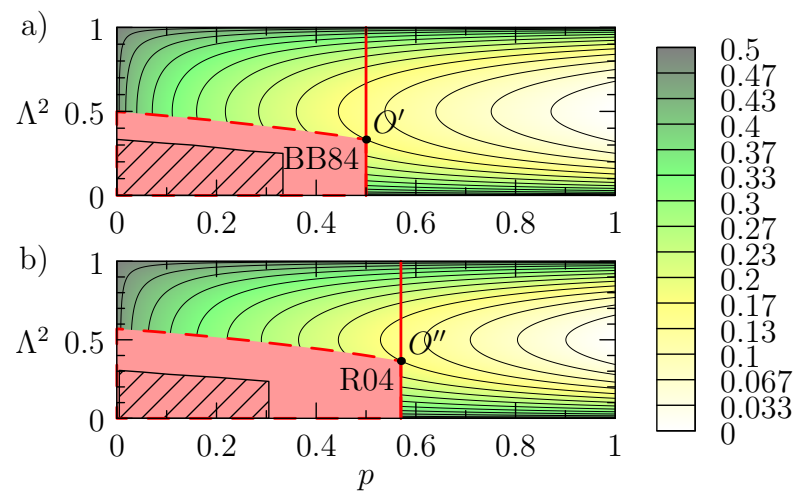

FIG. 3: (Color online) Cloning parameters, QBER and the cloning-attack security of the (a) BB84 and (b) R04 as a function of the cloning asymmetry parameter $p$ and cloning "strength" $\Lambda$. Dashed red lines show the QBER bound corresponding to the zero-length distilled key, i.e., $R=I_{A, B}-$ $\min \left(I_{A, E}, I_{B, E}\right)=0$. Thus, cloning enables successful eavesdropping in the regions marked by names of the QKDs. The optimal cloning attacks cause QBER $=16.7 \%$ if $p=0.57$ and $\Lambda^{2}=0.36$ (point $O^{\prime \prime}$ ) for R04 and $p=0.5$ and $\Lambda^{2}=0.33$ (point $O^{\prime}$ ) for BB84. The vertical solid red lines show the QBER bounds on the privacy of directly transmitted information corresponding to $I_{A, B}=I_{A, E}$, which are equal to $15.0 \%$ for R04 and $14.6 \%$ for BB84. The area of $\Lambda^{2} \geq 1 / 2\left(\Lambda^{2}=1 / 2\right)$ corresponds to the mirror phase-covariant OQC [22] (the asymmetric phase-covariant OQC [5, 25]). Hatched areas indicate the range of the cloning attacks without using quantum memory.

colinear type I degenerate spontaneous parametric downconversion process in $\mathrm{LiIO}_{3}$ crystal $(1 \mathrm{~cm}$ thick) pumped by a $\mathrm{cw} \mathrm{Kr}^{+}$laser beam $\left(\mathrm{TEM}_{00}\right.$ mode, $250 \mathrm{~mW}$ of optical power). Our source approximates two synchronized SPSs with the accuracy adequate for our demonstration, since the probability of having more than one photon in a mode for the 1 ns detection window is much lower than the probability of single-photon detection (approx. $10^{-} 5$ [20]). The emitted photons are in a separable polarization state; hence, Alice's state encoded as the signal does not change the polarization of the probe. Random choice of the states sent by Alice ensures that the polarization of the two photons is uncorrelated. This corresponds to having two independent but synchronized SPSs. However, in a real attack Eve would have to use a separate SPS. The photons propagate from the source to the OQC input via single-mode fibers. The photons are coherently superposed on the polarization-dependent beam splitter (PDBS). Next, the photons are subjected to polarization-sensitive filtering in both output modes (see BDA in Fig. 2). Finally, we postselect on coincidences - one photon in each of the two output modes of the cloner - and carry out polarization analysis of the two-photon state [28]. Using our tomographical data, we estimated the two-photon density matrix applying the maximum likelihood method [29]. We used the tomography results to numerically simulate Eve's attack assum- 
TABLE I: Performance of the OQC for BB84 and R04. The experimental values (subscript $E$ ) of the QBER and the secret-key rate $R$ calculated from the measured density matrices are compared with theoretical predictions (subscript $T$ ). The success probability $p_{s}$ of the OQC was estimated as in Ref. 1]. The OQC parameters $p$ and $\Lambda$ determine the shrinking of the Bloch sphere due to the cloning.

\begin{tabular}{llllll}
\hline \hline QKD & $R$ & QBER & $p_{s}$ & $p$ & $\Lambda^{2}$ \\
\hline $\mathrm{BB}_{4} 4_{T}$ & 0.00 & $16.7 \%$ & $13.7 \%$ & $1 / 2$ & $1 / 3$ \\
$\mathrm{BB} 84_{E}$ & $0.03 \pm 0.03$ & $18.5 \% \pm 1.5 \%$ & $15.1 \% \pm 1.1 \%$ & $1 / 2$ & $1 / 3$ \\
$\mathrm{R}_{4} 4_{T}$ & 0.00 & $16.7 \%$ & $12.7 \%$ & $4 / 7$ & $4 / 11$ \\
$\mathrm{R}_{E}$ & $0.01 \pm 0.08$ & $18.0 \% \pm 3.5 \%$ & $7.4 \% \pm 0.1 \%$ & $4 / 7$ & $4 / 11$ \\
\hline \hline
\end{tabular}

ing that she probes Alice's photon, keeps the probe until key sifting, and passes the probed photon to Bob (for details see Ref. [20]). We calculated the QBER and secretkey rate and compared them with theoretical predictions in Table I

The results indicate that our attack would be possible if QM was available. However, to deploy this device in a real QKD network, one has to consider several technological aspects of this attack. First, because of its probabilistic nature, the OQC introduces losses. The success probability of $10 \%-20 \%$ corresponds to $7-10 \mathrm{~dB}$ losses. Observing such losses might indicate that the line is insecure. Thus, Eve must mask these losses as usual channel losses. Supposing typical fibers losses of $3.5 \mathrm{~dB} / \mathrm{km}$ (as for the fibers in our experiment and in [1]), Eve would need to replace $2-3 \mathrm{~km}$ of the line with a fiber of negligible losses. Using photons at telecom wavelengths would be more practical than $826 \mathrm{~nm}$ light for communicating over large distances since, for the telecom-window wavelengths, the loses are $\sim 0.2 \mathrm{~dB} / \mathrm{km}$ [1, 2], which makes the distances about ten times larger and Eve's task more difficult. Typical detectors, designed for the telecom regime, provide low efficiency of about 0.25 (approx. $6 \mathrm{~dB}$ of losses) and high dark-count rate, i.e., noise. Much larger losses, which could enable eavesdropping, appear for long-range free-space transmission reaching, e.g., $157 \mathrm{~dB}$ for photons reflected from the Ajisai satellite [30]).

Eve should also control the unsuccessful cases when the signal and the probe propagate to Bob, who can detect them and raise alarm. Eve can achieve this by using quantum nondemolition (QND) measurement 31]. If she does not find a photon in her output mode, she will close the line towards Bob. Finally, Eve's attack relies on the perfect overlap between the signal and the probe photons (Hong-Ou-Mandel's interference). Typical full width at half maximum (FWHM) of photons generated via spontaneous parametric down-conversion corresponds to tens of $\mu \mathrm{m}$. Thus, the requirement on the two-photon overlap is of the order of $\mu \mathrm{m}$. This corresponds to a few fs. Any jitter caused by Alice leads to the reduced two-photon overlap, lower purity and fidelity of the output states. Eve can however overcome this by performing the QND detection at her OQC input, which triggers the cloning process but requires photon generation on demand. In a real cloning attack Eve has to prepare photons of the same spectral properties as the photons sent by Alice. In our experiment we use photons at $826 \mathrm{~nm}$ with spectral width FWHM=8.9 nm (160 fs coherence time). These are typical values reached by Alice using a femtosecond laser as a photon source. Let us note that the photon peaks need to overlap as perfectly as possible making the acceptable time difference corresponding to a fraction of coherence time which changes as wavelength squared divided by FWHM. Hence, overlapping is easier for longer wavelengths (e.g., in the telecom window) and narrow FWHM. Both the parameters should be tuned by Alice to maximize the security of the QKD.

Conclusions. - We investigated the feasibility of symmetric individual attacks on BB84 [10] and R04 [11] assuming that Eve tracks the key sifting and uses a multifunctional OQC [1]. We optimized quantum cloning such that the minimum mutual information between an eavesdropper and a legitimate user was equal to the mutual information between the legitimate users at the lowest QBER. Thus, legitimate users cannot distil a secret key from their raw key bits. Consequently we found tolerable QBER for this kind of attack to be $16.7 \%$ for BB84 and R04. We performed the proof-of-principle experiment in which $R \approx 0$ was attained for QBER $=18.5 \% \pm 1.5 \%$ for BB84 and QBER $=18.0 \% \pm 3.5 \%$ for R04. Our experiment together with the reported progress in development of QM (see, e.g., [14]) suggest that, even in the presence of SPSs and perfect detectors, the QKD could be successfully attacked with a probe similar to ours if Alice and Bob tolerate too high QBER (see Table \) or losses (approx. $7 \mathrm{~dB}$ for our device). Our experiment shows that the OQCs are interesting both from the fundamental and practical points of view as tools of quantum cryptanalysis as they establish the security bound for an important class of QKDs.

We thank Ravindra Chhajlany, Anirban Pathak, and Radim Filip for discussions. K.B. and A.M. were supported by Grant No. DEC-2011/03/B/ST2/01903 and No. DEC2011/02/A/ST2/00305 of the Polish National Science Centre. K.B. and K.L. were supported by Grants No. CZ.1.05/2.1.00/03.0058, No. CZ.1.07/ 2.3.00/20.0017, CZ.1.07/2.3.00/20.0058, CZ.1.07/2.3.00/30.0004 and No. CZ.1.07/2.3.00/30.0041, A.Č. and J.S. acknowledge support by the GACR Grant No. P205/ 12/0382.
[1] N. Gisin, G. Ribordy, W. Tittel, and H. Zbinden, "Quantum cryptography," Rev. Mod. Phys. 74, 145 (2002).
[2] T.-Y Chen et al., "Metropolitan all-pass and inter-city 
quantum communication network," Opt. Express 18, 27217 (2010).

[3] I. Georgescu and F. Nori, "Quantum technologies: an old new story," Phys. World 25, 16 (2012).

[4] W.-Y. Hwang, "Quantum key distribution with high loss: Toward global secure communication," Phys. Rev. Lett. 91, 057901 (2003).

[5] N.J. Cerf and J. Fiurášek, "Optical quantum cloning," Progress in Optics, edited by E. Wolf (Elsevier, Amsterdam, 2006), Vol. 49, p. 455.

[6] N. Gisin, S. Pironio, and N. Sangouard, "Proposal for implementing device-independent quantum key distribution based on a heralded qubit amplifier," Phys. Rev. Lett. 105, 070501 (2010).

[7] R. T. Glasser, H. Cable, J.P. Dowling, F. De Martini, F. Sciarrino, and C. Vitelli, "Entanglement-seeded, dual, optical parametric amplification: Applications to quantum imaging and metrology," Phys. Rev. A 78, 012339 (2008); N. Spagnolo, C. Vitelli, V.G. Lucivero, V. Giovannetti, L. Maccone, and F. Sciarrino, "Phase estimation via quantum interferometry for noisy detectors," Phys. Rev. Lett. 108, 233602 (2012); C. Vitelli, N. Spagnolo, L. Toffoli, F. Sciarrino, and F. De Martini, "Enhanced resolution of lossy interferometry by coherent amplification of single photons," Phys. Rev. Lett. 105, 113602 (2010).

[8] F. De Martini, F. Sciarrino, and C. Vitelli, "Entanglement test on a microscopic-macroscopic system," Phys. Rev. Lett. 100, 253601 (2008).

[9] P. Sekatski, N. Brunner, C. Branciard, N. Gisin, and C. Simon, "Towards quantum experiments with human eyes as detectors based on cloning via stimulated emission," Phys. Rev. Lett. 103, 113601 (2009).

[10] C. Bennett and G. Brassard, "Quantum cryptography: public key distribution and coin tossing" in Proceedings of the IEEE International Conference on Computers, Systems, and Signal Processing (IEEE, New York, 1984), p. 175 .

[11] J.M. Renes, "Spherical-code key-distribution protocols for qubits," Phys. Rev. A 70, 052314 (2004).

[12] L. Lydersen, C. Wiechers, C. Wittmann, D. Elser, J. Skaar, and V. Makarov, "Hacking commercial quantum cryptography systems by tailored bright illumination," Nat. Photon. 4, 686 (2010).

[13] I. Gerhardt, Q. Liu, A. Lamas-Linares, J. Skaar, C. Kurtsiefer, and V. Makarov, "Full-field implementation of a perfect eavesdropper on a quantum cryptography system" Nat. Commun. 2, 349 (2011).

[14] H. Specht, C. Nölleke, A. Reiserer, M. Uphoff, E. Figueroa, S. Ritter, and G. Rempe, "A single-atom quantum memory," Nature (London) 473, 190 (2011).

[15] N. Lütkenhaus, "Security against eavesdropping in quantum cryptography," Phys. Rev. A 54, 97 (1996).

[16] P.W. Shor and J. Preskill, "Simple Proof of Security of the BB84 Quantum Key Distribution Protocol," Phys. Rev. Lett. 85, 441 (2000).

[17] J.-C. Boileau, K. Tamaki, J. Batuwantudawe, R. Laflamme, and J.M. Renes, "Unconditional security of a three state quantum key distribution protocol," Phys. Rev. Lett. 94, 040503 (2005).

[18] C.A. Fuchs, N. Gisin, R.B. Griffiths, C.S. Niu, and A. Peres, "Optimal eavesdropping in quantum cryptography. I. Information bound and optimal strategy," Phys. Rev. A 56, 1163 (1997).

[19] N. Gisin and B. Huttner, "Quantum cloning, eavesdrop- ping, and Bell's inequality," Phys. Lett. A 228, 13 (1997).

[20] See Supplemental Material for technical details and extra figures.

[21] K. Lemr, K. Bartkiewicz, A. Černoch, J. Soubusta, and A. Miranowicz, "Experimental linear-optical implementation of a multifunctional optimal qubit cloner," Phys. Rev. A 85, 050307(R) (2012).

[22] K. Bartkiewicz, A. Miranowicz, and Ş. K. Özdemir, "Optimal mirror phase-covariant cloning," Phys. Rev. A 80, 032306 (2009).

[23] D. Bruß, M. Cinchetti, G.M. D'Ariano, and C. Macchiavello, "Phase-covariant quantum cloning," Phys. Rev. A 62, 012302 (2000).

[24] V. Bužek and M. Hillery, "Quantum copying: Beyond the no-cloning theorem," Phys. Rev. A 54, 1844 (1996).

[25] L. Bartůšková, M. Dušek, A. Černoch, J. Soubusta, and J. Fiurášek, "Fiber-optics implementation of an asymmetric phase-covariant quantum cloner," Phys. Rev. Lett. 99, 120505 (2007).

[26] A. Chefles, "Quantum state discrimination," Contemp. Phys. 41, 401 (2000).

[27] I. Csiszár and J. Körner, "Broadcast channels with confidential messages," IEEE Trans. Inf. Theory IT-24, 339 (1978).

[28] E. Halenková, A. Černoch, K. Lemr, J. Soubusta, and S. Drusová, "Experimental implementation of the multifunctional compact two-photon state analyzer," Appl. Opt. 51, 474 (2012).

[29] M. Ježek, J. Fiurášek, and Z. Hradil, "Quantum inference of states and processes," Phys. Rev. A 68, 012305 (2003).

[30] P. Villoresi et al., "Experimental verification of the feasibility of a quantum channel between space and Earth," New J. Phys. 10033038 (2008).

[31] M. Bula, K. Bartkiewicz, A. Černoch, and K. Lemr, "Entanglement-assisted scheme for nondemolition detection of the presence of a single photon," Phys. Rev. A 87, 033826 (2013). 


\section{Supplementary material:}

In this supplement we give more technical details on cloning transformations, cloning-based hacking and experimental data processing. We also compare graphically our theoretical and experimental tomographic results.

\section{THE CLONING MACHINE}

\section{A. Cloning transformation}

The general phase-covariant cloning transformation is given by

$$
\begin{aligned}
|0\rangle_{a} & \rightarrow \Lambda|000\rangle_{b, e, c}+\bar{\Lambda}\left(\sqrt{p}|01\rangle_{b, e}+\sqrt{q}|10\rangle_{b, e}\right)|1\rangle_{c} \\
|1\rangle_{a} & \rightarrow \Lambda|111\rangle_{b, e, c}+\bar{\Lambda}\left(\sqrt{p}|10\rangle_{b, e}+\sqrt{q}|01\rangle_{b, e}\right)|0\rangle_{c}
\end{aligned}
$$

in the Hilbert space of the two clones of Bob in mode $b$ and Eve in mode $e$ extended by an ancillary mode $c$. Moreover, 0 (1) denotes horizontal $H$ (vertical $V$ ) polarization, while $\bar{\Lambda}=\sqrt{1-\Lambda^{2}}$ and $p=1-q$ are positive real numbers. The mode $c$ has to be traced out to provide the state shared by Bob and Eve. The transformation (1) equally disturbs all the equatorial qubits,

$$
\left|a_{n}\right\rangle=\frac{1}{\sqrt{2}}\left[|0\rangle+\exp \left(i \phi_{n}\right)|1\rangle\right]
$$

where $n=0,1, \ldots$. We experimentally implemented the cloning transformation, given by Eq. (1), by fixing the values of $\Lambda$ and $p$, and by introducing polarization sensitive losses in the BDAs.

Eve, to make her cloning-based eavesdropping not easily detectable, ensures that 0 's and 1's are measured by Bob with the same probability. To achieve this, Eve uses one of the following transformations with probability $1 / 2$ :

$$
\begin{aligned}
& U_{0}\left|a_{n}, 0\right\rangle=C_{0}\left[\Lambda|00\rangle+\bar{\Lambda} \mathrm{e}^{i \phi_{n}}(\sqrt{q}|01\rangle+\sqrt{p}|10\rangle)\right], \\
& U_{1}\left|a_{n}, 1\right\rangle=C_{1}\left[\Lambda|11\rangle+\bar{\Lambda} \mathrm{e}^{i \phi_{n}}(\sqrt{q}|10\rangle+\sqrt{p}|01\rangle)\right],
\end{aligned}
$$

where $C_{x}=\sqrt{p_{x} / 2}$ with $p_{x}(x=0,1)$ being the success probability of the transformation $U_{x}$ including the transmission losses and the probability of having a single photon in each mode. Note that $p_{x}$ should not depend on $n$ since the cloning is phase-covariant, but because of experimental imperfections this is not always true. However, if $p_{x}$ does not change much with $n \mathrm{~m}$, which is the case of our cloner [1], one can replace it with the average over $n$. The transformations $U_{x}$ are implemented by overlapping the signal and probe photons on the polarizationdependent beam splitter and polarization-dependent filters. It is apparent that in general the resulting state shared by Bob and Eve is a mixed state. For convenience in the following sections we will use shorthand notation $\left|\psi_{x, n}\right\rangle=U_{x}\left|a_{n}, x\right\rangle$.

\section{B. Cloning parameters and polarization-sensitive losses}

In our implementation we randomly swap between $H$ and $V$ initial polarizations of the probe. If the probing photon is initially $H$-polarized then in order to set $\Lambda$ and $p$ we have to ensure the following transmittance ratios:

$$
\begin{aligned}
& \frac{\tau_{b, H}}{\tau_{b, V}}=\left(\frac{\Lambda}{\bar{\Lambda} \sqrt{p}}\right)^{2} \frac{(1-\mu)(1-\nu)}{(1-2 \mu)^{2}}, \\
& \frac{\tau_{e, H}}{\tau_{e, V}}=\left(\frac{\Lambda}{\bar{\Lambda} \sqrt{1-p}}\right)^{2} \frac{\mu \nu}{(1-2 \mu)^{2}},
\end{aligned}
$$

where $\tau_{x, y}$ stands for the intensity transmittance of the polarization $y=H, V$ in the spatial mode of Bob $(x=b)$ and Eve $(x=e) ; \mu$ and $\nu$ stand for the amplitude transmittance for the $H$ and $V$ polarizations of the polarization-dependent beam splitter. In the ideal case, the latter transmittances should be equal to $\mu=(1+1 / \sqrt{3}) / 2$ and $\nu=(1-1 / \sqrt{3}) / 2$. Due to manufacturing imperfections, the real transmittances are $\mu=0.77$ and $\nu=0.19$. If the probe is initially $V$ polarized, we fix

$$
\begin{aligned}
& \frac{\tau_{b, H}}{\tau_{b, V}}=\left(\frac{\bar{\Lambda} \sqrt{1-p}}{\Lambda}\right)^{2} \frac{(2 \nu-1)^{2}}{2(1-\mu)(1-\nu)}, \\
& \frac{\tau_{e, H}}{\tau_{e, V}}=\left(\frac{\bar{\Lambda} \sqrt{p}}{\Lambda}\right)^{2} \frac{(2 \nu-1)^{2}}{\mu \nu} .
\end{aligned}
$$

All the transmittances are set according to Eq. (5) in the corresponding BDAs.

\section{GAINING INFORMATION FROM A CLONE}

\section{A. The R04 protocol}

In the R04 protocol states sent by Alice and then measured by Bob read as

$$
\begin{aligned}
\left|a_{n}\right\rangle & =\frac{1}{\sqrt{2}}\left[|0\rangle+\exp \left(i \pi \frac{2 n}{3}\right)|1\rangle\right], \\
\left|b_{n}\right\rangle & =\frac{1}{\sqrt{2}}\left[|0\rangle+\exp \left(i \pi \frac{2 n+1}{3}\right)|1\rangle\right],
\end{aligned}
$$

where $n=0,1,2$. Let us assume that Bob announces that he did not measure state $\left|b_{n}\right\rangle$. If the protocol is continued that means that Alice sent $\left|a_{n}\right\rangle$ or $\left|a_{n \oplus 1}\right\rangle$, which corresponds to Alice obtaining bit value 1 or 0 , correspondingly. The bit value obtained by Bob depends on what he measured. If Bob measured $\left|b_{n \oplus 2}\right\rangle\left(\left|b_{n \oplus 1}\right\rangle\right)$, then his bit is $1(0)$. Since Eve waits for the Bob's announcement, she knows the number $n$. Moreover, she knows the initial state of her qubit $|x\rangle$ and by using this knowledge she performs the adequate POVM on her qubit. Eve designs POVMs $\left|e_{1}(x, n)\right\rangle\left\langle e_{1}(x, n)\right|\left(\left|e_{0}(x, n)\right\rangle\left\langle e_{0}(x, n)\right|\right)$ in advance in order to discriminate between Alice and $\mathrm{Bob}$ 
a)

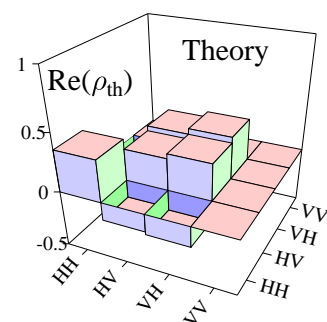

b)

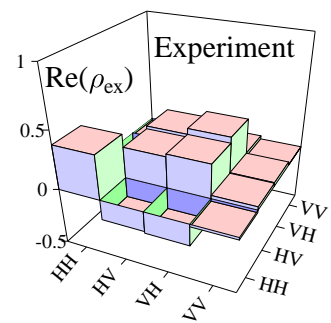

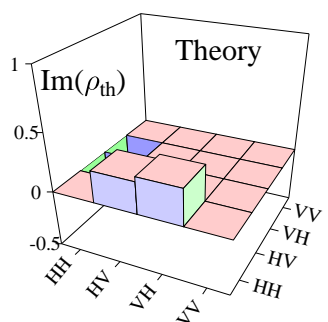

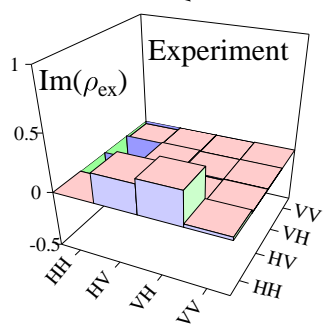

FIG. 4: (Color online) The reconstructed two-photon state $\rho_{\text {ex }}$ shared by Eve and Bob compared to the experimental prediction $\rho_{\text {th }}$ if Eve has probed $\left|a_{1}\right\rangle$ with the $H$-polarized photon in R04: (a) theory and (b) experiment. The fidelity of experimental matrix calculated as $F=\left(\operatorname{Tr} \sqrt{\sqrt{\rho_{\mathrm{th}}} \rho_{\mathrm{ex}} \sqrt{\rho_{\mathrm{th}}}}\right)^{2}$ is $98 \%$. The complete density matrix describing Bob's and Eve's photons is a mixture of density matrices for Eve using $V$ - and $H$-polarized photons.

a)

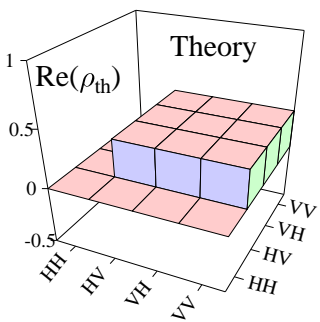

b)
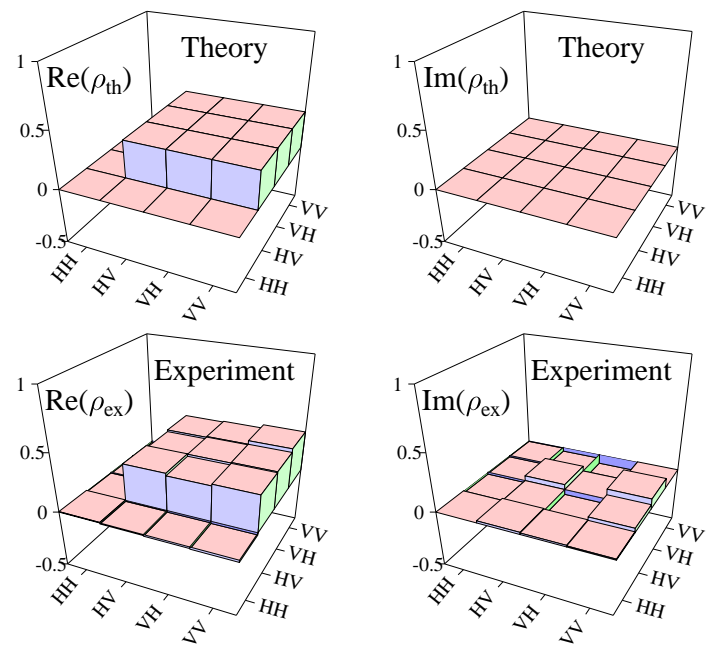

FIG. 5: (Color online) Same as in Fig. 4 but for BB84, where Eve has probed $\left|a_{0}\right\rangle$ with the $V$-polarized photon. Here, the fidelity of experimental matrix is $96 \%$.

both getting 1's (0's). The joint probability of obtaining bit values $k$ by Alice, $l$ by Bob, and $m$ by Eve is given by

$$
p_{x, n}(k, l, m)=N_{x, n}\left|\left\langle b_{n \oplus(1+l)}, e_{m}(x, n) \mid \psi_{x, n \oplus(1-k)}\right\rangle\right|^{2},
$$

where the normalization constant $N_{x, n}$ ensures that $\sum_{k, l, m} p_{x, n}(k, l, m)=1$. Therefore, the final probability distribution is given as

$$
p(k, l, m)=\frac{1}{6} \sum_{x=0}^{1} \sum_{n=0}^{2} p_{x, n}(k, l, m) .
$$

In order to calculate $\left|e_{m}(x, n)\right\rangle$ let us first introduce the following ancillary states

$$
\left|\epsilon_{m}(x, n)\right\rangle=\left\langle b_{n \oplus(1+m)} \mid \psi_{x, n \oplus(1-m)}\right\rangle,
$$

where $m=0,1$. For the ancillary states we can calculate the Bloch vectors as

$$
\vec{\epsilon}_{m}(x, n)=\left\langle\epsilon_{m}(x, n)|\vec{\sigma}| \epsilon_{m}(x, n)\right\rangle,
$$

where $\vec{\sigma}=\left(\sigma_{x}, \sigma_{y}, \sigma_{z}\right)$. Then, one can easily derive the Bloch vectors describing the Eve's POVMs as follows

$$
\begin{aligned}
\vec{e}_{m} & =\frac{\vec{\epsilon}_{m}-\vec{\epsilon}_{1-m}}{\left|\vec{\epsilon}_{m}-\vec{\epsilon}_{1-m}\right|} \\
& =\left(\sin \theta_{m} \cos \varphi_{m}, \sin \theta_{m} \sin \varphi_{m}, \cos \theta_{m}\right) .
\end{aligned}
$$

These measurement directions are known to be optimal for the state estimation and correspond to Helstrom's measurement. With the help of Eq. (11), Eve's POVMs are defined by the following pure states

$$
\left|e_{m}\right\rangle=\cos \frac{\theta_{m}}{2}|0\rangle+\mathrm{e}^{i \varphi_{m}} \sin \frac{\theta_{m}}{2}|1\rangle .
$$

Therefore, for the R04 protocol we are able to easily calculate the joint probability distribution of all the parties obtaining various bit values by knowing the state shared by Eve and Bob after the cloning transformation. In our experiment this state is obtained by the two-qubit polarization tomography (see Fig. (4).

\section{B. The BB84 protocol}

In the BB84 protocol states sent by Alice and measured by Bob read as

$$
\begin{aligned}
& \left|a_{n}\right\rangle=\frac{1}{\sqrt{2}}\left[|0\rangle+\exp \left(i \pi \frac{n}{2}\right)|1\rangle\right], \\
& \left|b_{n}\right\rangle=\frac{1}{\sqrt{2}}\left[|0\rangle+\exp \left(i \pi \frac{n}{2}\right)|1\rangle\right],
\end{aligned}
$$

where $n=0,1,2,3$. In case of the BB84 protocol Alice sends $\left|a_{0}\right\rangle$ and $\left|a_{2}\right\rangle$ for the first basis (e.g., $X$ basis), and $\left|a_{1}\right\rangle$ and $\left|a_{3}\right\rangle$ for the second basis (e.g., $Y$ basis). For the analyzed class of attacks, we assume that Eve knows the basis because she listens to the public announcements of Alice and Bob. Since Eve focuses only on the cases when the bases of Alice and Bob match, we can simply write the joint probability function as

$$
p_{x, y}(k, l, m)=N_{x, y}\left|\left\langle b_{2 l+y}, e_{m} \mid \psi_{x, 2 k+y}\right\rangle\right|^{2},
$$

where $N_{x, y}=1 / \sum_{k, l, m} p_{x, y}(k, l, m)$ and $x, y=0,1$ enumerate the initial state of Eve's qubit and the basis in the BB84 protocol, correspondingly. Therefore, the final probability distribution, which is used to calculate the mutual information between all the parties, is given as

$$
p(k, l, m)=\frac{1}{4} \sum_{x, y=0}^{1} p_{x, y}(k, l, m) .
$$


However, as in the case of the R04 protocol, we need to express $\left|e_{m}(x, y)\right\rangle$ in terms of the information available to Eve. The qubits obtained by Eve read as

$$
\left|\epsilon_{m}(x, y)\right\rangle=\left\langle b_{y+2 m} \mid \psi_{x, y+2 m}\right\rangle .
$$

Next, Eve constructs her POVMs analogously to the case of R04, i.e., by first using Eq. (11) and then Eq. (12).

\section{Experimental data processing}

Since, in our experiment, we automatically reconstruct a two-photon density matrix describing a pure state shared by Bob and Eve

$$
\rho_{x, n}=p_{x}^{-1}\left|\psi_{x, n}\right\rangle\left\langle\psi_{x, n}\right| .
$$

In our experiment the purity of the two reconstructed components is usually about $97 \%$ (see Figs. 4 and 5). We are able to calculate the probability distributions $p_{k, l, m}$ directly from the experimental data using Eqs. (8) and (15). This is because we can rewrite the expressions for the probabilities comprising the joint distributions using the density matrix formalism and the reconstructed matrices directly. Note that the expression for the joint probabilities implicitly assume that the success rate is uniform for all the configurations.

This success rate $p_{s}$, assumed to be uniform, is estimated in the first approximation by $p_{x}$ averaged over all the equatorial states used in the discussed protocols. In the experiment we obtain $p_{x}$ by comparing coincidence rate with and without applying the polarizationdependent beam splitter and polarization-dependent filters. Moreover, if Eve wants Bob's results to be symmetric with respect to swapping between the $H$ and $V$ polarizations, she should provide (if necessary by introducing additional losses) $p_{s}=p_{x=0}=p_{x=1}$. Alternatively, if $p_{x}$ varies little with $x$, Eve can do nothing and then $p_{s}$ can be approximated by $\left(p_{0}+p_{1}\right) / 2$. The success rates $p_{s}$ given in Tab. I were obtained by the following prescription.

The measured probability distributions $p(k, l, m) \equiv$ $p_{A, B, E}(k, l, m)$ for Alice, Bob, and Eve having various bit values are used to establish the real quantum bit error rate (QBER) for the both analyzed protocols as discussed in our Letter. However, for establishing the secret bit rate $R$ of the sifted key bits, first we determine the mutual Shannon information between the parties using the standard formula

$$
I_{X, Y}=I_{Y, X}=\sum_{x, y=0}^{1} p_{X, Y}(x, y) \log _{2}\left[\frac{p_{X, Y}(x, y)}{p_{X}(x) p_{Y}(y)}\right],
$$

where $p_{X, Y}(x, y)=\sum_{z=0}^{1} p_{X, Y, Z}(x, y, z)$ and $p_{X}(x)=$ $\sum_{y=0}^{1} p_{X, Y}(x, y)$ for $\{X, Y, Z\}=\{A, B, E\}$. Using Eq. (18) one can easily verify that the mutual information between Alice and Bob is simply given by $I_{A, B}=$ $1+\delta \log _{2} \delta+(1-\delta) \log _{2}(1-\delta)$ in terms of the probability $\delta$ of Bob obtaining a wrong bit value, which corresponds to the QBER.

Note that the cloning operation works with the success rate $p_{s}<1$ and for the remaining cases we do not detect any coincidences. Thus, for the particular initial state of the cloning machine, a fraction of $r=1-p_{s}$ of the photons sent by Alice is absorbed by the cloning machine. In our experiment this fraction is about $r \approx 80 \%$, which corresponds to about $7 \mathrm{~dB}$ of losses.

\section{SOURCE OF SINGLE PHOTONS}

In this section we demonstrate that the source of photons used in our experiments approximates a singlephoton source (SPS) to be used by Alice in QKD. The SPS is approximated by one of the modes of the downconverted light. In our experiment the intensity of the down-converted light is low to ensure that the probability of generating four (and larger numbers) of photons is negligible. The photon statistics of such downconverted beam is described by a thermal distribution $P(n)=\bar{n}^{n} /\left(1+\bar{n}^{n+1}\right)$ [2] with a small average number of photons $\bar{n} \ll 10^{-3}$ (we ensure that in the experiment). Thus, $P(n>1) \approx 0$ and we work with an approximate source of photon pairs $|1\rangle|1\rangle$, where each of the photons is in a different spatial mode.

Generating a photon in the first-order process is a random event. The down-conversion events appear independently of the number of down-converted photons $n$ in the time window of $1 \mathrm{~ns}$, thus the probability of having $n$ photons is given by Poisson's distribution:

$$
P(\lambda, n)=\lambda^{n} \exp (-\lambda) / n !,
$$

where $\lambda=\bar{n}$ is the average number of photons in the time window. We can calculate the probability of not detecting any photons in the $1 \mathrm{~ns}$ window directly from the number of detector clicks $N=35 \times 10^{3}$ that appeared as $P_{0}=1-N \times 10^{-9}=0.999965$. This is correct if the usual time interval between two detection events is larger than the dead time of the detectors corresponding to about $35 \mathrm{~ns}$. On the other hand, $P_{0}$ can be also calculated using a mathematical model of the ON/OFF (bucket) detectors of efficiency $\eta$ (in our experiment $\eta=0.5$ ) as

$$
P_{0}=\sum_{n=0}^{\infty} P(\lambda, n)(1-\eta)^{n},
$$

where the only unknown $\lambda$ is estimated numerically to be $\lambda=7.00 \times 10^{-5}$ assuming $P_{0}=0.999965$ and $\eta=0.5$. Therefore the probability of having more than one photon in the time window is equal to

$$
P_{>1}=1-\sum_{n=0}^{1} P\left(\lambda=7 \times 10^{-5}, n\right)=2.45 \times 10^{-9} .
$$


Moreover, since the probability of not having photons in the $35 \mathrm{~ns}$ dead time window is very high as it reaches $P^{35}\left(\lambda=7.00 \times 10^{-5}, n=0\right)=0.9976$, the above- presented reasoning provides results in good agreement with our other estimates.

[1] K. Lemr, K. Bartkiewicz, A. Černoch, J. Soubusta, and bridge University Press, Cambridge (2006).

[2] C. Gerry, P. Knight, Introductory Quantum Optics, Cam- 